\title{
Immunoprotective effects of oral intake of heat-killed Lactobacillus pentosus strain b240 in elderly adults: a randomised, double-blind, placebo-controlled trial
}

\author{
Shoji Shinkai ${ }^{1 *}$, Masamichi Toba ${ }^{2}$, Takao Saito ${ }^{2}$, Ikutaro Sato $^{2}$, Mina Tsubouchi $^{2}$, Kiyoto Taira ${ }^{2}$, \\ Keiji Kakumoto $^{2}$, Takashi Inamatsu ${ }^{3}$, Hiroto Yoshida ${ }^{1}$, Yoshinori Fujiwara ${ }^{1}$, Taro Fukaya ${ }^{1}$, \\ Tetsuya Matsumoto ${ }^{4}$, Kazuhiro Tateda ${ }^{5}$, Keizo Yamaguchi ${ }^{5}$, Noriyuki Kohda ${ }^{2}$ and Shigeru Kohno ${ }^{6}$ \\ ${ }^{1}$ The Research Team for Social Participation and Community Health, Tokyo Metropolitan Institute of Gerontology, \\ 35-2 Sakae-cho, Itabashi-ku, Tokyo 173-0015, Japan \\ ${ }^{2}$ Otsu Nutraceuticals Research Institute, Otsuka Pharmaceutical Company Limited, Otsu 520-0002, Japan \\ ${ }^{3}$ Tokyo Metropolitan Geriatric Hospital, Tokyo 173-0015, Japan \\ ${ }^{4}$ Tokyo Medical University, Tokyo 160-8402, Japan \\ ${ }^{5}$ Toho University School of Medicine, Tokyo 143-8540, Japan \\ ${ }^{6}$ Nagasaki University School of Medicine, Nagasaki 852-8501, Japan
}

(Submitted 19 April 2012 - Final revision received 26 July 2012 - Accepted 26 July 2012 - First published online 5 September 2012)

\section{Abstract}

Oral intake of Lactobacillus pentosus strain b240 (b240) has been shown to enhance the secretion of salivary secretory IgA in elderly adults. However, its clinical benefits remain to be determined. We tested the hypothesis that b240 exerts a protective effect against the common cold in elderly adults. The design of the present study was a randomised, double-blind, placebo-controlled trial (RCT) with parallel threegroup comparison. For this purpose, 300 eligible elderly adults were randomly allocated to one of three groups, namely a placebo, lowdose or high-dose b240 group. Participants in the low-dose and high-dose b240 groups were given tablets containing $2 \times 10^{9}$ or $2 \times 10^{10}$ cells, respectively, of heat-killed b240, while those in the placebo group were given tablets without b240. Each group consumed their respective tablets once daily for 20 weeks. The common cold was assessed on the basis of a diary. Change in quality of life was evaluated using the SF- $36{ }^{\circledR}$. Of the total participants, 280 completed the 20 -week RCT. The accumulated incidence rate of the common cold was $47 \cdot 3$, 34.8 and $29.0 \%$ for the placebo, low-dose b240 and high-dose b240 groups, respectively $(P$ for trend $=0 \cdot 012)$. Lower incidence rates were consistently observed throughout the experimental period in the b240 groups (log-rank test, $P=0 \cdot 034$ ). General health perception, as determined by the SF-36 ${ }^{\circledR}$, dose-dependently increased in the b240 groups ( $P$ for trend $=0 \cdot 016$ ). In conclusion, oral intake of b240 significantly reduced the incidence rate of the common cold in elderly adults, indicating that b240 might be useful in improving resistance against infection through mucosal immunity.

Key words: Common cold: Elderly adults: Lactobacillus: Immunoprotection

Infectious diseases are a leading cause of mortality and significant morbidity in elderly adults, who are at greater risk than younger populations ${ }^{(1)}$. Increasing age has been associated with diminished humoral and cell-mediated immunity against newly encountered pathogens or vaccines ${ }^{(2-6)}$, which suggests a need for countermeasures against agerelated immune dysfunctions. Secretory IgA (SIgA) secretion in saliva decreases with age ${ }^{(7-11)}$, and this reduction might increase the risk of respiratory infections. Therefore, slowing the age-related decline in salivary SIgA secretion may help improve the health of elderly adults.
To date, many lactic acid bacteria have been shown to modulate specific and/or non-specific immune responses in animal models and occasionally in human subjects ${ }^{(12)}$. Certain species enhance IgA secretion/concentration in the gut of mice $^{(13)}$, the faeces of healthy children ${ }^{(14)}$ and the saliva of infants $^{(15)}$. However, there are no studies on increasing salivary SIgA secretion in elderly adults. Lactobacillus pentosus strain b240 (b240) is an anaerobic, non-sporulating, Gram-positive bacterium originally isolated from fermented tea leaves ${ }^{(16)}$. In our laboratory, we have determined that, among 150 strains of lactic acid bacteria, b240 has the highest

Abbreviations: b240, Lactobacillus pentosus strain b240; FAS, full analysis set; PPS, per-protocol set; QOL, quality of life; RCT, randomised, double-blind, placebo-controlled trial; SIgA, secretory IgA; URT, upper respiratory tract.

* Corresponding author: Dr S. Shinkai, fax +8133579 4776, email sshinkai@tmig.or.jp 
IgA-inducing potency in mouse Peyer's patch cells ${ }^{(17)}$. In addition, we found that oral intake of heat-killed b240 (corresponding to $2 \times 10^{9}$ or $2 \times 10^{10}$ cell counts/d for 3 weeks) significantly increased salivary IgA secretion and non-significantly increased the amount of faecal IgA in younger adults ${ }^{(18)}$.

On the basis of these findings, we have recently conducted a randomised, double-blind, placebo-controlled trial (RCT) and observed that oral intake of heat-killed b240 for 12 weeks was effective in enhancing salivary SIgA secretion in elderly adults ${ }^{(19)}$. Mean salivary SIgA secretion in a b240 group steadily increased until week 4 (when it was $20 \%$ higher than at week 0) and then remained stable until week 12. Changes in SIgA secretion over the intervention period were significantly greater in a b240 group than in a placebo group $^{(19)}$. These results strongly suggest the potential effectiveness of b240 in improving mucosal immunity and resistance against infection.

It is now necessary to explore the clinical benefits of b240. Does enhancement of mucosal immunity by b240 actually protect infection in elderly adults? The specific objective of the present study was to test the hypothesis that b240 exerts a protective effect against the common cold in elderly adults. In addition, we examined whether b240 increased quality of life (QOL).

\section{Experimental methods}

\section{Study participants}

Participants were recruited via a local advertisement for adults aged 65 years or older who resided in Tokyo or its suburbs. Before giving consent, they were given a detailed explanation of the purpose of the study and the potential risks of participation. Of the 558 individuals who submitted consent forms, 538 participated in the first-phase screening test (see the Assessment section). The initial exclusion criteria were as follows: (1) current smoker; (2) vigorous (more than six metabolic equivalents) exerciser; (3) participants with nonstandard values on blood chemistry, blood pressure or pulse; (4) participants with hepatitis, cancer, inflammatory bowel diseases, rheumatoid arthritis or other diseases affecting the digestive tract or immune system; (5) participants with chronic obstructive lung diseases such as asthma and chronic bronchitis, allergic rhinitis with any medication and a past history of pneumonia; (6) participants with periodontitis or haemorrhagic stomatitis; (7) participants taking antibiotics; (8) participants taking antiflatulents, antidiarrhoeals, steroids, immunosuppressive drugs or other drugs related to the activation or suppression of the digestive or immune systems; (9) participants declared ineligible for participation by a medical doctor. During testing, ninety-nine out of the 538 participants were found to meet at least one of the exclusion criteria and were excluded. The remaining 439 individuals underwent a second-phase screening test during which they visited the study site (Tokyo Metropolitan Institute of Gerontology) twice on two different days a week apart and provided a total of four saliva samples (two samples per $\mathrm{d} \times 2 \mathrm{~d}$ ), which were used to determine SIgA levels. Saliva collection and the method for measuring SIgA were identical to previously described methods ${ }^{(19)}$. On the basis of saliva test results, 300 individuals with relatively low $\mathrm{CV}$ in SIgA secretion rate were selected for the subsequent RCT (Fig. 1).

\section{Sample size}

We calculated sample size on the assumption that a Japanese adult has an average of two common colds per year and that the use of b240 would result in a $25 \%$ reduction in the incidence of the common cold. Assuming a power of $80 \%$ and a significance level of 0.05 , we estimated that we would need 100 persons per group and per session (20 weeks) to show a $25 \%$ difference between the groups.

\section{Study design}

The experiment was conducted from March to July 2010 as a RCT with parallel three-group comparison. To this end, 300 subjects were randomly allocated to one of three groups, i.e. a placebo group, low-dose b240 group or high-dose b240 group, matched by age and sex. Investigators and participants remained blinded to treatment assignment until the end of the study. The participants in the low-dose and high-dose b240 groups were given tablets containing $2 \times 10^{9}$ or $2 \times 10^{10}$ cells, respectively, of heat-killed b240, while those in the placebo group were given tablets without containing b240. They were instructed to consume one tablet per $\mathrm{d}$ at breakfast for 20 weeks. The participants were also instructed to record in a diary all upper respiratory tract (URT) or other symptoms experienced and any medication taken during the 20 -week intervention. They visited the study site at weeks $0,2,4,8$, 12,16 and 20 to be interviewed by physicians and to undergo assessment.

\section{Lactobacillus pentosus strain b240 and placebo tablets}

A trial tablet containing heat-killed b240 was prepared as follows: after cultivating b240 and performing repeated washing, the cells were sterilised in an autoclave for $15 \mathrm{~min}$ and freeze-dried. The non-viable b240 was counted with a flow cytometer (Beckman Coulter Epics XL-MCL; Beckman Coulter K. K.) and adjusted to produce a b240 tablet with $2 \times 10^{9}$ or $2 \times 10^{10}$ cells. A tablet without b240 was used as the placebo. The three tablet types were indistinguishable in size, taste and appearance.

\section{Assessment}

During the first-phase screening, all participants ( $n$ 538) were assessed with respect to their physical characteristics (height, weight and BMI), blood pressure, urine and blood profiles, oral health, medical history, living arrangement, vaccination history and history of the common cold. For this purpose, trained personnel asked participants about their medical history, including chronic obstructive lung diseases and allergic rhinitis, medications, and experience with flu vaccination 


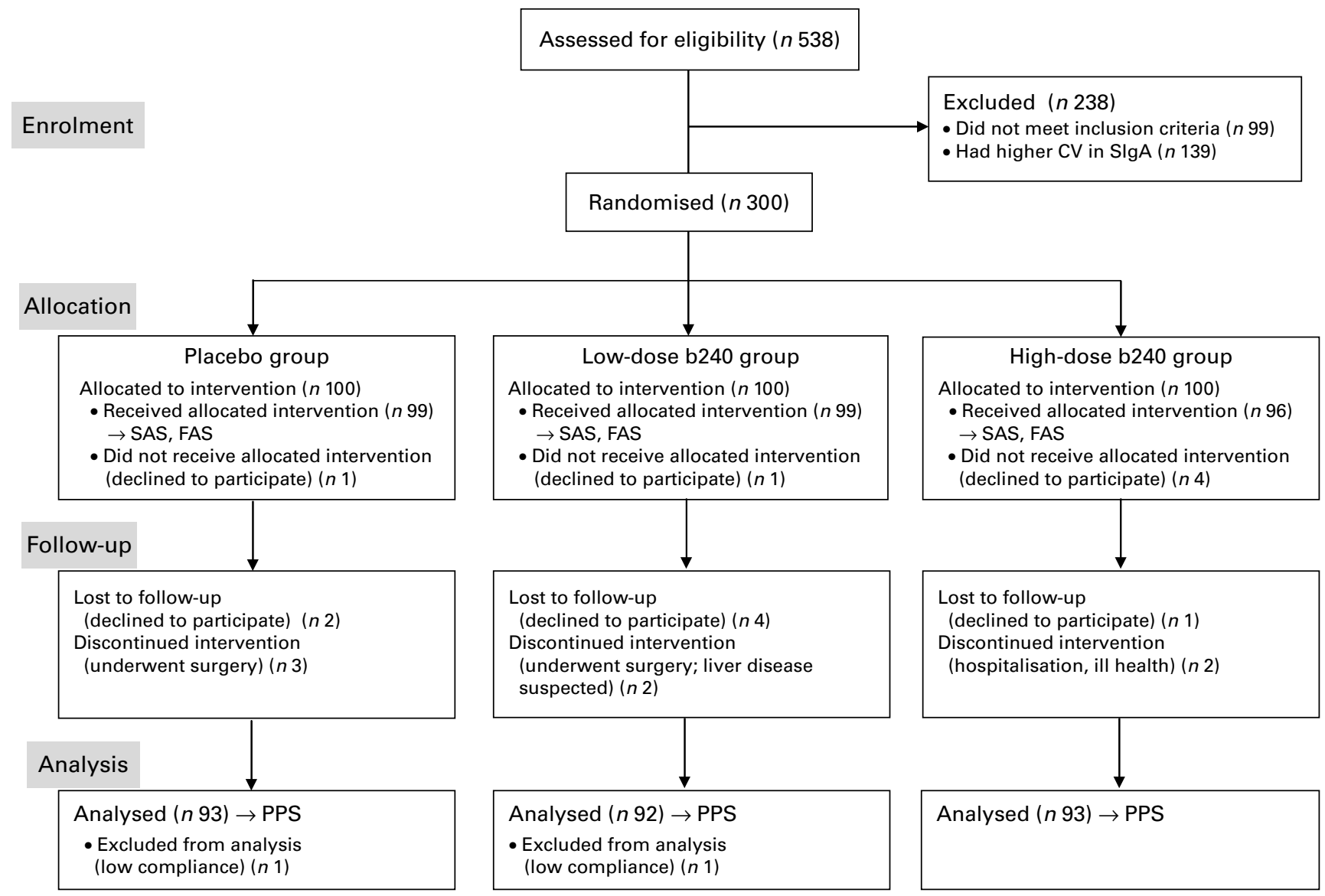

Fig. 1. Study flow and subjects. SIgA, secretory IgA; SAS, safety analysis set; FAS, full analysis set; PPS, per-protocol set.

and the common cold in the previous year. All subjects underwent urinalysis and blood testing and were examined for periodontitis and haemorrhagic stomatitis by a dental hygienist. We used this information as the pre-intervention data for the 300 RCT-eligible subjects. Additionally, the 300 subjects were assessed for stress and mood, nutritional intake and physical activity by answering the Profile of Mood States Questionnaire $^{(20)}$, the Brief Dietary Habit Questionnaire ${ }^{(21,22)}$ and the Japan Arteriosclerosis Longitudinal Study Physical Activity Questionnaire ${ }^{(23)}$, respectively, at week 0.

After the intervention (at week 20), those who completed the RCT ( $n$ 280) were reassessed for all the items mentioned above, except physical characteristics, blood pressure, urine profile and oral health. From the subjects' diaries, we collected information on protocol compliance, occurrence of adverse symptoms and events, and medication used throughout the intervention period.

\section{Blood testing}

Blood was collected from participants in the first-phase screening ( $n$ 538) and those who completed the 20-week RCT study ( $n$ 280) at pre-intervention (at the first-phase screening) and post-intervention (at week 20), respectively. Samples were tested for circulating cell counts (erythrocytes, leucocytes and platelets), $\mathrm{Hb}$, haematocrit, serum total protein, albumin, albumin:globulin ratio, total cholesterol, TAG, NEFA, fasting glucose, $\mathrm{Hb} \mathrm{A}_{1 \mathrm{c}}$, aspartate aminotransferase, alanine aminotransferase, $\gamma$-glutamyl transpeptidase, alkaline phosphatase, lactate dehydrogenase, choline esterase, total bilirubin, direct and indirect bilirubin, urea $\mathrm{N}$, uric acid, creatinine, $\mathrm{Na}, \mathrm{Cl}, \mathrm{K}, \mathrm{Ca}$ and inorganic $\mathrm{P}$ by standardised procedures at the Special Reference Laboratory, Inc., Tokyo, Japan.

\section{Common cold}

The common cold was assessed on the basis of diaries in which each participant was instructed to record any upper respiratory symptoms and body temperature. Body temperature was measured twice per d soon after waking up in the morning and before bedtime at night, using a thermometer (Digital thermometer C203; TERUMO Company). Based on comprehensive criteria for common cold assessment derived from combination cold remedies in Japan ${ }^{(24,25)}$, the Centers for Disease Control overview ${ }^{(26)}$ and the Jackson cold scale ${ }^{(27)}$, we selected the following twelve items as symptoms of the common cold: (1) runny nose, (2) stuffy nose, (3) sneezing, (4) sore throat, (5) cough and (6) sputum, together with systemic symptoms including (7) headache, (8) chills, 
(9) malaise, (10) body aches, (11) fever and (12) watery eyes. Subjects used a four-level scale ('none', 'mild', 'moderate' or 'severe', corresponding to scores of $0,1,2$ or 3 , respectively) to rate the severity of each of these symptoms daily for 20 weeks. The presence or absence of each symptom and its severity, and body temperature were recorded in their diaries and the records were mailed to the study site weekly. Once a week, a nurse contacted each subject by telephone to confirm the content of the diary. The overall score was calculated by adding the severity scores for individual cold symptoms, and day-to-day scores were plotted on a graph to visualise changes in symptoms over time (score graph). Subjects visited the study site at weeks $0,2,4,8,12,16$ and 20 to be interviewed by a physician, who confirmed the content of the diary and determined whether the subject sought treatment for a cold at a neighbourhood clinic after the previous visit.

Common cold assessment was performed in three steps. First, based on the criteria for common cold assessment from combination cold remedies in Japan (two or more URT symptoms) ${ }^{(24)}$, we established the following criteria for a candidate cold: (1) two or more URT symptoms (upper respiratory symptoms included runny nose, stuffy nose, sneezing, sore throat, cough and sputum) or (2) one URT symptom plus one or more systemic symptoms (systemic symptoms included headache, chill, malaise, body pain, fever and watery eyes). Fever was regarded as present only when body temperature either in the morning or in the evening increased by more than $0.5^{\circ} \mathrm{C}$ from the corresponding average body temperature throughout the experimental period. Second, using the score graph, we examined whether candidate cold events met any combination criteria according to the duration of disease and necessary condition. If any episode lasted for 2-14d, it was automatically defined as a candidate common cold. If any symptom persisted longer than $15 \mathrm{~d}$, the corresponding score was replaced by 0 . Finally, an expert panel comprising four physicians who were specialised in infectious diseases reviewed each case of the common cold and made the final determination.

\section{Quality of life}

Subject QOL was assessed at week 0 and week 20 after the start of the intervention using the SF- $36 \mathrm{v} 2^{\mathrm{TM}}$ questionnaire (Standard Japanese Version; Institute for Health Outcomes \& Process Evaluation Research). Changes in QOL were evaluated for eight domains of the SF-36 by subtracting baseline scores at week 0 from those at week 20 .

\section{Assessment of safety}

We compared adverse health symptoms/events that occurred during the intervention period and blood profiles before and after the intervention in the placebo and b240 groups.

\section{Statistical analysis}

All measured data were presented as means and standard deviations. Comparisons among the placebo and b240 groups with respect to age, BMI, influenza vaccination and common cold in the previous year, basal SIgA secretion, nutritional intake, physical activity and stress assessment were analysed by one-way ANOVA, the Cochran-Mantel-Haenszel test or the $\chi^{2}$ test. For the accumulated incidence of the common cold during the 20-week period, dose dependence was tested with the Cochran-Armitage test, and Steel's multiple comparison test was used for inter-group comparison. The log-rank test was used for inter-group comparison of Kaplan-Meier estimates of common cold incidence over the course of 20 weeks. For multiple comparison, the log-rank test with the closed testing procedure ( $v$. placebo) was used.

Dunnett's multiple comparison test was performed for the inter-group comparison of the QOL score. To examine whether the effect of b240 on the QOL score was independent of common cold experience, we used generalised linear models in which we entered changes in score $(\Delta$ post - pre) as the target variable, and group (placebo to high dose) and common cold experience (yes or no) as explanatory variables.

The paired $t$ test was used for the intra-group comparison of blood variables, pre- and post-intervention. A two-tailed $P$ value less than 0.05 was considered to indicate statistical significance in all tests. Data were analysed using SAS software (version 9.1; SAS Institute Japan Limited).

\section{Ethical considerations}

The present study was carried out in compliance with the study protocol, the ethical principles of the Declaration of Helsinki, the Ethical Guidelines on Epidemiological Studies (Ministry of Education, Culture, Sports, Science and Technology, and Ministry of Health, Labour and Welfare, Japan), and the Personal Information Protection Law. The research protocol was approved by the Ethics Committee of Tokyo Metropolitan Institute of Gerontology (15 January 2010). Written informed consent was obtained from all subjects.

\section{Results}

\section{Study flow}

Fig. 1 shows the study flow diagram. Of the 538 elderly persons who were assessed for eligibility, 300 eligible individuals were enrolled in the RCT. Before the start of the experiment, six subjects declined to participate due to personal reasons. Thus, the safety analysis set and full analysis set (FAS) included 294 subjects, among whom 280 completed the 20week RCT study. We further excluded two subjects because of low compliance: one had a test tablet intake rate of $87 \cdot 2 \%$ (criterion, $\geq 90 \%$ ); the other had consumed prohibited food items (i.e. fermented foods) for $126 \mathrm{~d}$ in total (criterion, within four consecutive weeks). Thus, 278 subjects were analysed in the efficacy analysis as the per-protocol set (PPS).

\section{Subject characteristics}

The clinical features of the participants in the RCT, pre- and post-intervention, are shown in Table 1 . There were no

\footnotetext{
deviations. Comparisons among the placebo and b240
} 
Table 1. Characteristics of the subjects who completed the 20-week randomised, double-blind, placebo-controlled trial (per-protocol set)

(Mean values and standard deviations; number of subjects and percentages)

\begin{tabular}{|c|c|c|c|c|c|c|c|c|c|c|c|c|c|c|}
\hline & \multicolumn{7}{|c|}{ Pre-intervention } & \multicolumn{7}{|c|}{ Post-intervention } \\
\hline & \multicolumn{2}{|c|}{ Placebo } & \multicolumn{2}{|c|}{ Low-dose b240 } & \multicolumn{2}{|c|}{ High-dose b240 } & \multirow[b]{2}{*}{$P$} & \multicolumn{2}{|c|}{ Placebo } & \multicolumn{2}{|c|}{ Low-dose b240 } & \multicolumn{2}{|c|}{ High-dose b240 } & \multirow[b]{2}{*}{$P^{*}$} \\
\hline & Mean & SD & Mean & SD & Mean & SD & & Mean & SD & Mean & SD & Mean & SD & \\
\hline Subjects $(n)$ & \multicolumn{2}{|c|}{93} & \multicolumn{2}{|c|}{92} & \multicolumn{2}{|c|}{93} & 0.875 & & & & & & & \\
\hline Male & \multirow{2}{*}{\multicolumn{2}{|c|}{46}} & \multirow{2}{*}{\multicolumn{2}{|c|}{44}} & \multirow{2}{*}{\multicolumn{2}{|c|}{48}} & & & & & & & & \\
\hline Female & & 47 & 48 & & 45 & & & & & & & & & \\
\hline Age (years) & 70.9 & 3.8 & $71 \cdot 0$ & 4.0 & 70.8 & 3.4 & 0.901 & & & & & & & \\
\hline $\mathrm{BMI}\left(\mathrm{kg} / \mathrm{m}^{2}\right)$ & $22 \cdot 8$ & $2 \cdot 7$ & $22 \cdot 8$ & $2 \cdot 8$ & 23.8 & $2 \cdot 7$ & 0.023 & $22 \cdot 6$ & $2 \cdot 7$ & $22 \cdot 7$ & $2 \cdot 8$ & $23 \cdot 5$ & $2 \cdot 7$ & 0.042 \\
\hline Living with child under 12 years old & & & \multirow{2}{*}{\multicolumn{2}{|c|}{1}} & & & 0.413 & & & & & & & \\
\hline$n$ & \multicolumn{2}{|c|}{4} & & & & & & & & & & & & \\
\hline$\%$ & & & & & & & & & & & & & & \\
\hline Influenza vaccination in the past year & & & & & & & 0.917 & & & & & & & \\
\hline$n$ & & & & & & & & & & & & & & \\
\hline$\%$ & & & & & & & & & & & & & & \\
\hline Common cold in the past year & & & & & & & 0.256 & & & & & & & \\
\hline 0 times & & & & & & & & & & & & & & \\
\hline$n$ & & & & & & & & & & & & & & \\
\hline$\%$ & & & & & & & & & & & & & & \\
\hline 1 time & & & & & & & & & & & & & & \\
\hline$n$ & & & & & & & & & & & & & & \\
\hline$\%$ & & & & & & & & & & & & & & \\
\hline 2 times & & & & & & & & & & & & & & \\
\hline$n$ & & & & & & & & & & & & & & \\
\hline$\%$ & & & & & & & & & & & & & & \\
\hline 3 times or over & & & & & & & & & & & & & & \\
\hline$n$ & & & & & & & & & & & & & & \\
\hline$\%$ & & & & & & & & & & & & & & \\
\hline SlgA secretion rate $(\mu \mathrm{g} / 5 \mathrm{~min})$ & 305 & 144 & 304 & 130 & 296 & 147 & 0.794 & & & & & & & \\
\hline Dietary intake & & & & & & & & & & & & & & \\
\hline Energy $(\mathrm{kJ} / \mathrm{d})$ & 8388 & 2471 & 8035 & 2482 & 8675 & 2575 & 0.224 & 8699 & 3029 & 7954 & 2435 & 8431 & 2536 & 0.262 \\
\hline Protein $(\mathrm{g} / \mathrm{d})$ & 79 & 24 & 76 & 26 & 84 & 31 & 0.155 & 84 & 34 & 79 & 31 & 81 & 25 & 0.456 \\
\hline Lipid (g/d) & 64 & 20 & 60 & 22 & 65 & 23 & 0.241 & 60 & 25 & 56 & 21 & 58 & 19 & 0.372 \\
\hline Carbohydrate $(\mathrm{g} / \mathrm{d})$ & 250 & 78 & 241 & 84 & 256 & 67 & 0.380 & 260 & 96 & 245 & 79 & 261 & 84 & 0.373 \\
\hline Physical activity (MET/d) & $34 \cdot 8$ & 4.0 & $35 \cdot 1$ & $4 \cdot 2$ & 34.9 & 4.6 & 0.892 & $35 \cdot 2$ & 3.8 & 35.5 & 4.5 & $35 \cdot 3$ & 0.5 & 0.876 \\
\hline POMS & & & & & & & & & & & & & & \\
\hline Tension-anxiety & 49 & 8 & 46 & 7 & 47 & 8 & 0.034 & 48 & 8 & 48 & 10 & 47 & 9 & 0.745 \\
\hline Depression-dejection & 52 & 12 & 49 & 10 & 49 & 10 & 0.106 & 51 & 11 & 50 & 10 & 49 & 10 & 0.574 \\
\hline Anger-hostility & 50 & 8 & 48 & 8 & 49 & 8 & 0.377 & 50 & 8 & 49 & 8 & 49 & 9 & 0.775 \\
\hline Vigour & 53 & 8 & 51 & 9 & 52 & 8 & 0.249 & 51 & 8 & 50 & 9 & 51 & 9 & 0.523 \\
\hline Fatigue & 49 & 9 & 47 & 8 & 46 & 7 & 0.052 & 52 & 10 & 51 & 9 & 51 & 11 & 0.745 \\
\hline Confusion & 50 & 10 & 48 & 9 & 49 & 10 & 0.432 & 51 & 9 & 50 & 10 & 50 & 10 & 0.713 \\
\hline
\end{tabular}

b240, Lactobacillus pentosus strain b240; SIgA, secretory Ig A; MET, metabolic equivalents; POMS, Profile of Mood States.

${ }^{*} \chi^{2}$ test for sex distribution and living with child under 12 years old, Cochran-Mantel-Haenszel test for the common cold in the past year and one-way ANOVA for all other data. 
Table 2. Incidence, total episode, duration and severity of the common cold during the 20-week intervention

(Mean values and standard deviations; number of subjects and percentages)

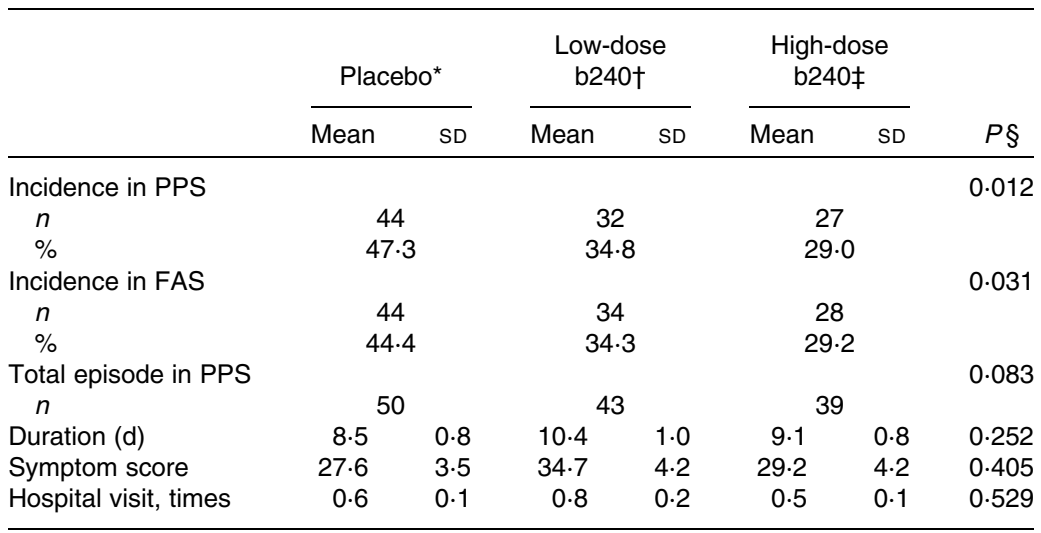

b240, Lactobacillus pentosus strain b240; PPS, per-protocol set; FAS, full analysis set.

${ }^{*}$ PPS, $n$ 93; FAS, $n 99$.

†PPS, $n$ 92; FAS, $n 99$

‡PPS, $n$ 93; FAS, $n 96$.

$\S$ Cochran-Armitage test for incidence and total episode, and ANOVA for independent samples with log-transformed values of other variables.

significant differences among the three groups in age, influenza vaccination and common cold in the previous year, basal salivary SIgA secretion, dietary intake and energy expenditure, or stress.

\section{Incidence of the common cold}

Table 2 shows a comparison of the accumulated incidence rates and other variables of the common cold in the PPS and FAS during the 20-week intervention period among the three groups. There was a significant b240 dose-dependent decrease in the incidence of the common cold in both the PPS and FAS ( $P$ for trend $=0.012$ for PPS and $P$ for trend $=0.031$ for FAS). Multiple comparison testing showed that the high-dose group had a significant $(P=0.020)$ and a marginally significant $(P=0.051)$ lower incidence rate when

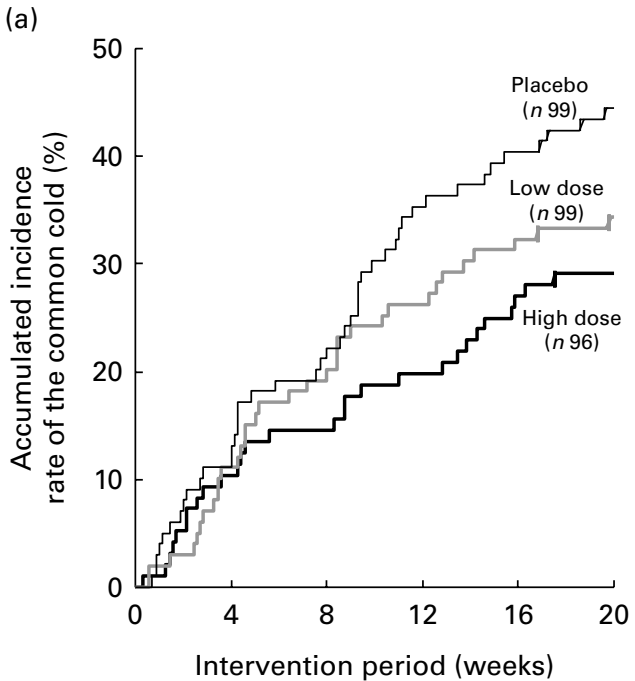

compared with the corresponding placebo group in the PPS and FAS, respectively. There was also a marginally significant difference in the total number of cold episodes among the three groups ( $P$ for trend $=0.083$ ). However, mean duration, symptom score and hospital visits due to the common cold did not differ among the groups.

Fig. 2 illustrates the accumulated incidence rate of the common cold over the duration of the trial. The differences among the three groups were consistent throughout the trial in both the PPS and FAS (log-rank test: $\chi^{2} 6.74$ and $P=0.034$ for PPS; $\chi^{2} 4.99$ and $P=0.083$ for FAS). The log-rank test with the closed testing procedure showed that only the high-dose group had significantly $(P=0.012$ and 0.029$)$ lower rates over the trial period when compared with the corresponding placebo group in the PPS and FAS, respectively.

(b)

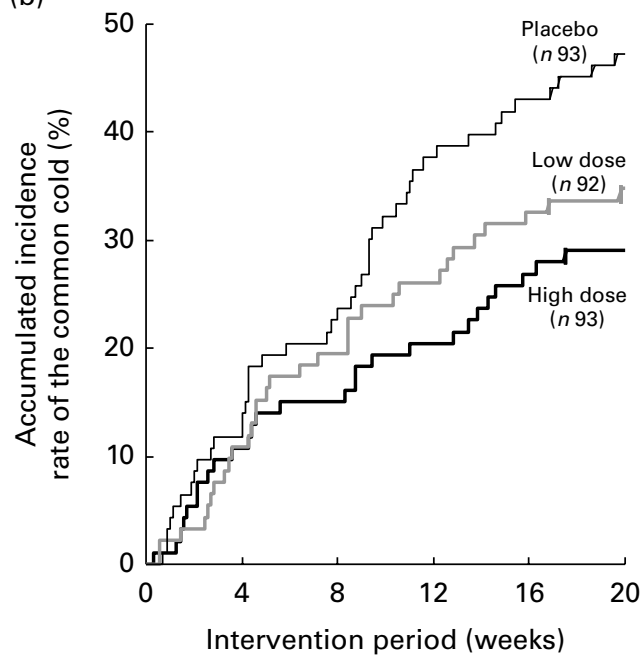

Fig. 2. Accumulated incidence rate of the common cold during the 20-week intervention. (a) Full analysis set (log-rank test: $\chi^{2}=4.99$ and $P=0.083$ ) (b) Per-protocol set (log-rank test: $\chi^{2}=6.74$ and $P=0.034$ ). 
Table 3. Changes in quality of life as assessed by the SF-36 (per-protocol set)

(Mean values and standard deviations)

\begin{tabular}{|c|c|c|c|c|c|c|c|c|c|}
\hline \multirow[b]{3}{*}{ Subscales of the SF-36 } & \multirow[b]{3}{*}{ Groups } & \multirow[b]{3}{*}{$n$} & \multicolumn{7}{|c|}{ NBS score } \\
\hline & & & \multicolumn{2}{|c|}{$\begin{array}{c}\text { Pre- } \\
\text { intervention }\end{array}$} & \multicolumn{2}{|c|}{$\begin{array}{c}\text { Post- } \\
\text { intervention }\end{array}$} & \multicolumn{2}{|c|}{$\Delta(\text { post }- \text { pre })^{*}$} & \multirow[b]{2}{*}{$P \dagger$} \\
\hline & & & Mean & SD & Mean & SD & Mean & SD & \\
\hline \multirow[t]{3}{*}{ Physical functioning } & Placebo & 93 & $51 \cdot 4$ & $7 \cdot 3$ & $50 \cdot 3$ & $9 \cdot 6$ & $-1 \cdot 1$ & $7 \cdot 1$ & Reference \\
\hline & Low-dose b240 & 92 & $52 \cdot 1$ & 5.9 & $50 \cdot 9$ & $7 \cdot 8$ & $-1 \cdot 2$ & $7 \cdot 1$ & 0.99 \\
\hline & High-dose b240 & 93 & $51 \cdot 2$ & $10 \cdot 4$ & $49 \cdot 7$ & $9 \cdot 3$ & -1.5 & $8 \cdot 1$ & 0.89 \\
\hline \multirow[t]{3}{*}{ Role physical } & Placebo & 93 & $52 \cdot 0$ & $7 \cdot 3$ & 49.5 & 11.0 & $-2 \cdot 4$ & $10 \cdot 8$ & Reference \\
\hline & Low-dose b240 & 92 & $52 \cdot 1$ & $6 \cdot 3$ & $50 \cdot 4$ & 8.8 & -1.6 & 7.6 & 0.78 \\
\hline & High-dose b240 & 93 & 51.6 & 8.7 & 50.4 & 9.4 & $-1 \cdot 2$ & $9 \cdot 1$ & 0.56 \\
\hline \multirow[t]{3}{*}{ Body pain } & Placebo & 93 & $51 \cdot 0$ & $8 \cdot 3$ & $51 \cdot 0$ & 9.9 & 0.0 & $9 \cdot 6$ & Reference \\
\hline & Low-dose b240 & 92 & 53.7 & 8.7 & $52 \cdot 2$ & 11.4 & -1.5 & $9 \cdot 1$ & 0.48 \\
\hline & High-dose b240 & 93 & $52 \cdot 0$ & 8.9 & 51.9 & 9.9 & -0.1 & 8.8 & 1.00 \\
\hline \multirow[t]{3}{*}{ General health perception } & Placebo & 93 & 54.9 & $7 \cdot 8$ & 53.4 & $8 \cdot 6$ & -1.4 & $7 \cdot 4$ & Reference \\
\hline & Low-dose b240 & 92 & 54.3 & $7 \cdot 2$ & 54.9 & $9 \cdot 6$ & 0.7 & 8.2 & $0 \cdot 11$ \\
\hline & High-dose b240 & 93 & 55.5 & $7 \cdot 8$ & $56 \cdot 6$ & $8 \cdot 2$ & $1 \cdot 1$ & 6.5 & 0.04 \\
\hline \multirow{3}{*}{ Vitality } & Placebo & 93 & $57 \cdot 1$ & 7.4 & $55 \cdot 1$ & $9 \cdot 6$ & $-2 \cdot 0$ & 8.4 & Reference \\
\hline & Low-dose b240 & 92 & $57 \cdot 8$ & 7.9 & 55.4 & 8.7 & $-2 \cdot 4$ & $7 \cdot 2$ & 0.92 \\
\hline & High-dose b240 & 93 & 57.9 & $7 \cdot 1$ & 55.9 & 9.5 & $-2 \cdot 0$ & $6 \cdot 7$ & 1.00 \\
\hline \multirow[t]{3}{*}{ Social functioning } & Placebo & 93 & 52.4 & $7 \cdot 0$ & $52 \cdot 1$ & $8 \cdot 0$ & -0.3 & $10 \cdot 2$ & Reference \\
\hline & Low-dose b240 & 92 & 53.6 & 6.9 & 53.1 & $7 \cdot 0$ & -0.6 & $8 \cdot 2$ & 0.98 \\
\hline & High-dose b240 & 93 & 52.5 & $6 \cdot 8$ & $51 \cdot 1$ & $8 \cdot 6$ & -1.4 & 9.7 & 0.67 \\
\hline \multirow[t]{3}{*}{ Role emotional } & Placebo & 93 & $52 \cdot 7$ & $6 \cdot 9$ & $51 \cdot 3$ & $9 \cdot 7$ & -1.5 & $10 \cdot 0$ & Reference \\
\hline & Low-dose b240 & 92 & 53.6 & $5 \cdot 2$ & 51.4 & 8.4 & $-2 \cdot 1$ & 6.8 & 0.82 \\
\hline & High-dose b240 & 93 & 52.5 & 7.9 & $51 \cdot 2$ & $9 \cdot 8$ & -1.3 & 7.9 & 0.98 \\
\hline \multirow{3}{*}{ Mental health } & Placebo & 93 & 54.9 & $7 \cdot 1$ & $55 \cdot 1$ & $7 \cdot 3$ & 0.2 & 7.6 & Reference \\
\hline & Low-dose b240 & 92 & $56 \cdot 0$ & 8.4 & $55 \cdot 0$ & $8 \cdot 7$ & $-1 \cdot 0$ & $7 \cdot 2$ & 0.40 \\
\hline & High-dose b240 & 93 & 55.8 & $7 \cdot 1$ & 55.7 & $7 \cdot 2$ & -0.1 & $6 \cdot 3$ & 0.93 \\
\hline
\end{tabular}

\section{Change in quality of life}

Table 3 shows a comparison of pre- and post-intervention QOL scores. From pre- to post-intervention, the score for the general health perception subscale in the b240 groups significantly improved when compared with that in the placebo group ( $P$ for trend=0.041). The generalised linear models showed that the improved general health perception in the b240 groups was independent of common cold experience during the intervention (placebo $v$. high-dose b240, adjusted $P$ value with Dunnett's multiple comparison test, $P=0.025$ ).

\section{Safety}

There was no apparent pattern in adverse health events during the intervention period among the placebo and b240 groups (data not shown). Likewise, there was also no differing change in any blood variables from pre- to post-intervention among the three groups (data not shown).

\section{Discussion}

In the present RCT, we found that oral intake of heat-killed b240 significantly dose-dependently reduced the incidence of the common cold in elderly adults. The accumulated incidence rate at week 20 was 29.0 and $34.8 \%$ for the high-dose and low-dose groups, respectively, while that for the placebo group was $47.3 \%$. The incidence rates in the b240 groups were consistently lower throughout the trial period. To our knowledge, the present study is the first to show that oral intake of heat-killed lactic acid bacteria dose-dependently reduces the incidence rate of the common cold in elderly adults.

Many investigators have studied the therapeutic and preventive effects of yogurt and other fermented dairy products containing lactic acid bacteria on diseases and disorders such as cancer, infection, gastrointestinal disorders and asthma ${ }^{(28)}$. Among these studies, several have reported that continuous intake of fermented products containing certain strains of lactic acid bacteria modulates the incidence, severity and/or duration of the common cold ${ }^{(29-33)}$. For example, the effects of probiotic strains Lactobacillus rhamnosus GG, Lactobacillus johnsonii La1, Lactobacillus casei DN-114001, Lactobacillus bulgaricus OLL1073R-1 and L. casei Shirota have been examined in children attending day care centres, enterally fed elderly, free-living elderly, community-dwelling elderly and athletes, respectively. Although the strain of lactic acid bacteria, target population, study design and sample size differed among these studies, they all have consistently reported slight to moderate protective effects against the common cold.

When compared with earlier studies, the present study has several unique strengths. First, we used heat-killed, non-viable b240. Although the presence of lactic acid bacteria in 
fermented products has been regarded as essential to their immunostimulatory effects, it is also believed that nonbacterial components, such as whey protein, short peptide and conjugated linoleic acid, contribute to the beneficial effects of these products ${ }^{(28)}$. Thus, we believe that the present study adds to the literature because it shows that the bacterial constituents of b240 have an essential role in immunostimulation. Second, because the present study was an RCT with parallel three-group comparison, we were able to examine the dose-response relationship. The incidence of the common cold was reduced by 39 and $26 \%$ in the high-dose and low-dose b240 groups, respectively. These values are substantially higher than corresponding estimates reported in previous studies, indicating that b240 has greater potency in stimulating mucosal immunity. Third, the elderly participants were recruited from general older populations and were highly compliant, with minimum withdrawal. This enabled us to extend the results to the general elderly population. Finally, the main outcome of the present study, i.e. occurrence of the common cold, was strictly defined according to a three-step procedure, which minimised the misclassification of the common cold. According to our records, about $5 \%$ of the total number of common cold episodes was directly checked on-site, and self-reported information was well validated by a physician.

Furthermore, the present study is the first to show that oral intake of lactic acid bacteria resulted in improved perception of general health among elderly adults. Of particular interest is that improved general health perception in the b240 groups was independent of common cold experience during the intervention. This finding suggests that b240 benefits other aspects of health and warrants further study.

What are the mechanisms underlying the immunoprotective effect of oral intake of b240? Mucosal immunity at the surface of the URT undoubtedly has a major role in resistance against the common cold. Adaptive humoral immune defence at mucosal surfaces is largely mediated by SIgA, the predominant Ig class in human external secretion ${ }^{(34)}$. Adaptive humoral mucosal immune responses are mainly initiated in an inductive site (e.g. Peyer's patch in the intestine). Sensitised mucosal immunocytes (e.g. $\operatorname{IgA}^{+} \mathrm{B}$ cells) then leave the inductive site, travel through the lymph, enter the circulation and migrate to diffuse mucosal effector sites (e.g. lamina propria), where they differentiate into memory or effector cells (e.g. IgAproducing plasma cells $)^{(35,36)}$. SIgA in saliva has been used as an indicator of mucosal immunity ${ }^{(37)}$. The salivary glands are the most important source of SIgA in the URT ${ }^{(38)}$. A lack of non-specific SIgA at the mucosal surface or an inability to produce specific SIgA can increase the risk of infection ${ }^{(39)}$

To date, we have demonstrated the b240 enhancement of IgA production from Peyer's patch cells in the mouse gut and elevations in IgA in salivary IgA secretion in younger adults after an oral intake of heat-killed b240 (corresponding to $2 \times 10^{9}$ or $2 \times 10^{10}$ cell counts/d for 3 weeks). Further, oral intake of heat-killed b240 for 12 weeks effectively enhanced salivary SIgA secretion in the elderly ${ }^{(19)}$. Taken together, these findings suggest the possibility that oral intake of b240 has an immunoprotective effect against the common cold via enhancement of salivary SIgA production after the activation of the gut mucosal immune system.

Although yogurt and fermented dairy products containing lactic acid bacteria have long been regarded to be beneficial for host defence mechanisms, the components responsible for these effects, and the mechanism by which these components exert their immunological modification, are not completely understood. However, lactic acid bacteria seem to have multipotent immunostimulatory effects and thus could stimulate varying aspects of human immune function. Therefore, the SIgA pathway may not be the sole mechanism linking b240 intake and immunoprotection against the common cold. Since the present study did not monitor immunological parameters over the course of the trial, the precise immune mechanism remains to be determined in future studies.

In terms of adverse health events and the blood profile, there was no significant difference among the three groups. Indeed, there was no noteworthy adverse event in any group. L. pentosus has a long history of use in cooked and uncooked fermented foods. Viable and heat-killed nonviable preparations of b240 were fully evaluated for shortterm and subchronic toxicity, and genotoxic potential; no toxic effects were detected ${ }^{(40)}$. In summary, continuous intake of b240 is safe over a wide dose range in humans.

A limitation of the present study warrants mention. The potential effects of b240 did not extend to the severity or duration of the common cold, perhaps because enhanced mucosal immunity may be protective against virus invasion but is ineffective once the infection is established. It is also possible that the severity and/or duration of the common cold might be substantially confounded by medication or hospital visits. A well-designed study is needed to examine the potential effect of b240 in moderating infection severity.

In summary, oral intake of heat-killed b240 significantly reduced the incidence of the common cold in elderly adults, and improved their perception of their general health. Although the immunological mechanism for this effect remains to be determined, the present results highlight the potential effectiveness of b240 in resistance against infection through mucosal immunity. To date, L. pentosus strains have been isolated from many fermented food items including dairy products ${ }^{(41-43)}$. Both the European Food Safety Authority and the International Dairy Federation classify L. pentosus as a safe agent for food use $\mathrm{e}^{(44,45)}$. Thus, the present study suggests that $L$. pentosus has the potential in contributing to human health and nutrition.

\section{Acknowledgements}

We thank Professor Sanae Okada, Faculty of Applied Bioscience, Tokyo University of Agriculture for providing us with lactic acid bacteria and Professor Matsumura, Kiryu University for group assignment and statistical analysis. We also thank the members of the Research Team for Social Participation and Health Promotion, Tokyo Metropolitan Institute of Gerontology, Otsu Nutraceuticals Research Institute, and Saga Nutraceuticals Research Institute, Otsuka Pharmaceutical Company Limited for their expert assistance. 
This study was conducted under the contract of the Tokyo Metropolitan Institute of Gerontology (TMIG) and Otsuka Pharmaceutical Company Limited. The present study received no specific grant from any funding agency in the public or not-for-profit sectors. The authors' contributions were as follows: S. S., M. Toba and N. K. contributed to all aspects of the study and the preparation of the manuscript; T. S., I. S. and $\mathrm{K}$. Taira were involved in the conception and design, the acquisition of the subjects and the data collection; M. Tsubouchi, H. Y., Y. F. and T. F. were responsible for the acquisition of the subjects and the data collection; K. K. performed the analysis and contributed to the interpretation of the data; T. I., T. M., K. Tateda, K. Y. and S. K. participated in the conception and design, and the interpretation of the data. All authors declare that there are no conflicts of interest.

\section{References}

1. Gavazzi G \& Krause KH (2002) Ageing and infection. Lancet Infect Dis 2, 659-666.

2. Phair J, Kauffman CA, Bjornson A, et al. (1978) Failure to respond to influenza vaccine in the aged: correlation with B-cell number and function. J Lab Clin Med 92, 822-828.

3. Cook JM, Gualde N, Hessel L, et al. (1987) Alterations in the human immune response to hepatitis $\mathrm{B}$ vaccine among the elderly. Cell Immunol 109, 89-96.

4. Burns EA, Lum LG, L'Hommedieu G, et al. (1993) Specific humoral immunity in the elderly: in vivo and in vitro response to vaccination. J Gerontol 48, B231-B236.

5. Kolibab K, Smithson SL, Shrine AK, et al. (2005) Immune response to pneumococcal polysaccharides 4 and 14 in elderly and young adults. I. Antibody concentrations, avidity and functional activity. Immun Ageing 2, 10.

6. Aspinall R, Del Giudice G, Effros RB, et al. (2007) Challenges for vaccination in the elderly. Immun Ageing $\mathbf{4}, 9$.

7. Smith DJ, Joshipura K, Kent R, et al. (1992) Effect of age on immunoglobulin content and volume of human labial gland saliva. J Dent Res 71, 1891-1894.

8. Challacombe SJ, Percival RS \& Marsh PD (1995) Age-related changes in immunoglobulin isotypes in whole and parotid saliva and serum in healthy individuals. Oral Microbiol Immunol 10, 202-207.

9. Miletic ID, Schiffman SS, Miletic VD, et al. (1996) Salivary IgA secretion rate in young and elderly persons. Physiol Behav 60, 243-248.

10. Evans P, Der G, Ford G, et al. (2000) Social class, sex, and age differences in mucosal immunity in a large community sample. Brain Behav Immun 14, 41-48.

11. Tanida T, Ueta E, Tobiume A, et al. (2001) Influence of aging on candidal growth and adhesion regulatory agents in saliva. J Oral Pathol Med 30, 328-335.

12. Ezendam J \& van Loveren H (2006) Probiotics: immunomodulation and evaluation of safety and efficacy. Nutr Rev 64, 1-14.

13. Park JH, Um JI, Lee BJ, et al. (2002) Encapsulated Bifidobacterium bifidum potentiates intestinal IgA production. Cell Immunol 219, 22-27.

14. Fukushima Y, Kawata Y, Hara H, et al. (1998) Effect of a probiotic formula on intestinal immunoglobulin A production in healthy children. Int J Food Microbiol 42, 39-44.

15. Tormo Carnicer R, Infante Pina D, Rosello Mayans E, et al. (2006) Intake of fermented milk containing Lactobacillus casei DN-114 001 and its effect on gut flora. Ann Pedatr (Barc) 65, 448-453.

16. Okada S, Daengsubha W, Uchimura T, et al. (1986) Flora of lactic acid bacteria in Miang produced in northern Thailand. J Gen Appl Microbiol 32, 57-65.

17. Yamahira S, Toba M, Kishi K, et al. (2006) Stimulation of mucosal immune system by lactic acid bacteria originating in tea. Jpn J Lactic Acid Bact 17, 57-60.

18. Kishi K, Kotani Y, Yamahira S, et al. (2006) Lactobacillus plantarum ONRIC b0240 enhanced salivary IgA in healthy adult volunteers. Jpn J Lactic Acid Bact 17, 132-137.

19. Kotani Y, Shinkai S, Okamatsu H, et al. (2010) Oral intake of Lactobacillus pentosus strain b240 accelerates salivary immunoglobulin A secretion in the elderly: a randomized, placebo-controlled, double-blind trial. Immun Ageing 7, 11.

20. Yokoyama K, Araki S, Kawakami N, et al. (1990) Production of the Japanese edition of Profile of Mood States (POMS): assessment of reliability and validity. Jpn J Public Health 37, 913-918.

21. Sasaki S, Yanagibori R \& Amano K (1998) Self-administered diet history questionnaire development for health education: a relative validation of the test-version by comparison with 3-day diet record in women. $J$ Epidemiol 8, 203-215.

22. Sasaki S, Ushio F, Amano K, et al. (2000) Serum biomarkerbased validation of a self-administered diet history questionnaire for Japanese subjects. J Nutr Sci Vitaminol 46, $285-296$.

23. Naito Y, Harada A, Inoue S, et al. (2003) Report of the physical activity research of the Japan Arteriosclerosis Longitudinal Study. Res Exerc Epidemiol 5, 1-7.

24. Takasaki Y, Maruyama N, Uchida T, et al. (2004) Clinical study of CR-22T for common cold syndrome. Prog Med 24, 1121-1131.

25. Yoshitsugu M \& Takigawa H (2010) Usefulness of SKC-036 in patients with common cold syndrome. Jpn Pharmacol Ther 38, 603-612.

26. Centers for Disease Control and Prevention (2011) Common cold and runny nose: overview of common cold. http:// www.cdc.gov/getsmart/antibiotic-use/URI/colds.html

27. Jackson GG, Dowling HF \& Muldoon RL (1962) Acute respiratory diseases of viral etiology. VII. Present concepts of the common cold. Am J Public Health Nations Health 52, 940-945.

28. Meydani SN \& Ha W-K (2000) Immunologic effects of yogurt. Am J Clin Nutr 71, 861-872.

29. Hatakka K, Savilahti E, Ponka A, et al. (2001) Effect of long term consumption of probiotic milk on infections in children attending day care centres: double blind, randomised trial. BMJ 322, 1-5.

30. Fukushima Y, Miyaguchi S, Yomano T, et al. (2007) Improvement of nutritional status and incidence of infection in hospitalised, enterally fed elderly by feeding of fermented milk containing probiotic Lactobacillus jobnsonii La1 (NCC533). Br J Nutr 98, 969-977.

31. Guillemard E, Tondu F, Lacoin F, et al. (2010) Consumption of a fermented dairy product containing the probiotic Lactobacillus casei DN-114001 reduces the duration of respiratory infections in the elderly in a randomised controlled trial. $\mathrm{BrJ}$ Nutr 103, 58-68.

32. Makino S, Ikegami S, Kume A, et al. (2010) Reducing the risk of infection in the elderly by dietary intake of yoghurt fermented with Lactobacillus delbrueckii ssp. bulgaricus OLL1073R-1. Br J Nutr 104, 998-1006.

33. Gleeson M, Bishop NC, Oliveira M, et al. (2011) Daily probiotic's (Lactobacillus casei Shirota) reduction of infection incidence in athletes. Int J Sport Nutr Exerc Metab 21, 55-64. 
34. Woof JM \& Mestecky J (2005) Mucosal immunoglobulins, Immunol Rev 206, 64-82.

35. Holmgren J \& Czerkinsky C (2005) Mucosal immunity and vaccines. Nat Med 11, Suppl. 4, S45-S53.

36. Brandtzaeg P (2009) Mucosal immunity: induction, dissemination, and effector functions. Scand I Immunol $\mathbf{7 0}$, 505-515.

37. Albers R, Antoine JM, Bourdet-Sicard R, et al. (2005) Markers to measure immunomodulation in human nutrition intervention studies. Br J Nutr 94, 452-481.

38. Tomasi TB (1992) The discovery of secretory IgA and the mucosal immune system. Immunol Today 13, 416-418.

39. Hanson LA, Björkander J \& Oxelius VA (1983) Selective IgA deficiency. In Primary and Secondary Immunodeficiency Disorders, pp. 62-64 [RK Chandra, editor]. Edinburgh: Churchill Livingstone.

40. Szabo NJ, Dolan LC, Burdock GA, et al. (2011) Safety evaluation of Lactobacillus pentosus strain b240. Food Chem Toxicol 49, 251-258.

41. Robert H, Gabriel V \& Fontagné-Faucher C (2009) Biodiversity of lactic acid bacteria in French wheat sourdough as determined by molecular characterization using speciesspecific PCR. Int J Food Microbiol 30, 53-59.

42. Tanasupawat S, Pakdeeto A, Thawai C, et al. (2007) Identification of lactic acid bacteria from fermented tea leaves (miang) in Thailand and proposals of Lactobacillus thailandensis sp. nov., Lactobacillus camelliae sp. nov., and Pediococcus siamensis sp. nov. J Gen Appl Microbiol 53, 7-15.

43. Kostinek M, Specht I, Edward VA, et al. (2007) Characterization and biochemical properties of predominant lactic acid bacteria from fermenting cassava for selection as starter cultures. Int J Food Microbiol 20, 342-351.

44. European Food Safety Authority (2011) Scientific opinion on the maintenance of the list of QPS biological agents intentionally added to food and feed (2011 update). EFSA $J$ 9, 2497-2578, http://www.efsa.europa.eu/en/efsajournal/doc/ 2497.pdf

45. Mogensen G, Salminen S \& O'brien J, et al. (2002) Inventory of microorganisms with a documented history of use in food Bulletin-International Dairy Federation, 10-19 International Dairy Federation, Brussels. 